\title{
Retention of Endogenous Viable Cells Enhances the Anti-Inflammatory Activity of Cryopreserved Amnion
}

\author{
Yi Duan-Arnold, ${ }^{1, *}$ Alexandra Gyurdieva, ${ }^{1}$ Amy Johnson, \\ Thomas E. Uveges, ${ }^{1}$ Douglas A. Jacobstein, ${ }^{1,2}$ and Alla Danilkovitch ${ }^{1}$ \\ ${ }^{1}$ Osiris Therapeutics, Inc., Columbia, Maryland. \\ ${ }^{2}$ ThermoFisher Scientific, Inc., Portage, Michigan.
}

Objective: Human amniotic membrane (hAM) has been used to treat wounds for more than 100 years. However, widespread use of fresh hAM has been limited due to its short shelf life and safety concerns. To overcome these concerns, different preservation methods have been introduced. The majority of these methods result in devitalized hAM (dev-hAM). Recently, we developed a cryopreservation method that retains all hAM components intact (int-hAM), including viable endogenous cells. To understand the advantages of retaining viable cells in preserved hAM, we compared the anti-inflammatory properties of int-hAM and dev-hAM.

Approach: The tissue composition of int-hAM and dev-hAM was compared with fresh hAM through histology and cell viability analysis. We also evaluated the ability of int-hAM and dev-hAM to regulate tumor necrosis factor- $\alpha$ (TNF- $\alpha$ ), interleukin-1 $\alpha$ (IL- $1 \alpha$ ), and IL-10 release when co-cultured with immune cells; to produce prostaglandin E2 (PGE2) on TNF- $\alpha$ stimulation; and to inhibit proteases.

Results: Int-hAM maintained the structural and cellular integrity of fresh hAM. Int-hAM had $>80 \%$ cell viability post-thaw and remained viable for at least a week in culture. Viable cells were not detected in dev-hAM. Compared with devhAM, int-hAM showed significantly greater downregulation of TNF- $\alpha$ and IL- $1 \alpha$, upregulation of PGE2 and IL-10, and stronger inhibition of collagenase.

Innovation and Conclusion: A new cryopreservation method has been developed to retain all native components of hAM. For the first time, we show that viable endogenous cells significantly augment the anti-inflammatory activity of cryopreserved hAM.

\section{INTRODUCTION}

CHRONIC WOUNDS OFTEN stall in the inflammatory stage and are characterized by high levels of proinflammatory cytokines and proteases. Inflammation and protease activity are critical for normal wound healing. However, elevated and pro- longed expression of inflammatory cytokines and proteases leads to the degradation of extracellular matrix (ECM) and growth factors, which prevents wounds from progressing into the proliferative and remodeling phases and inhibits healing. ${ }^{1}$ Therefore, wound-care modalities that ad-

(C) Yi Duan-Arnold, et al. 2015; Published by Mary Ann Liebert, Inc. This Open Access article is distributed under the terms of the Creative Commons Attribution Noncommercial License (<http:// creativecommons.org/licenses/by-nc/4.0/>) which permits any noncommercial use, distribution, and reproduction in any medium, provided the original author(s) and the source are credited.

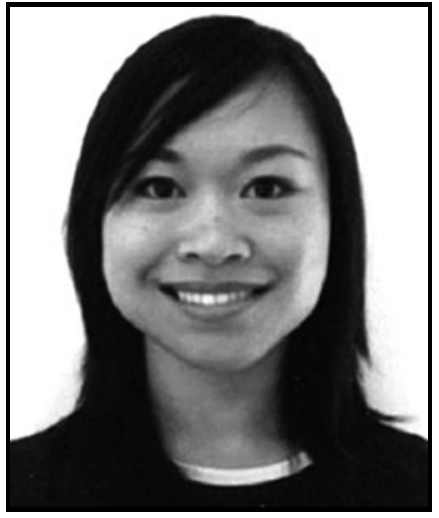

Yi Duan-Arnold, PhD

Submitted for publication January 26, 2015. Accepted in revised form March 23, 2015

*Correspondence: Osiris Therapeutics, Inc., 7015 Albert Einstein Drive, Columbia, MD 21046 (e-mail: yarnold@osiris.com). 
dress excessive inflammation have the potential to restart the healing process.

Human amniotic membrane (hAM) has been used for the treatment of wounds since $1910 .^{2}$ The low immunogenicity of hAM permits its allogeneic use. ${ }^{3}$ It acts as a physical barrier against bacterial contamination and also creates a moist environment required for healing. ${ }^{4}$ Furthermore, it reduces pain and has anti-inflammatory, anti-fibrotic, and anti-microbial activities that are beneficial for wound healing. ${ }^{5}$ Despite these benefits, the widespread use of fresh tissue has been limited due to its short shelf life and the risk of disease transmission associated with insufficient time for testing. ${ }^{6}$ Therefore, different preservation methods have been developed to overcome these limitations. These processing methods, however, alter the composition of hAM (endogenous cells, ECM, and growth factors) to varying degrees, which can have a negative impact on its functionality. ${ }^{7,8}$

Cryopreservation is one of the common preservation methods used for hAM. It has been reported that cryopreserved hAM better retains structural integrity and shows functional superiority in comparison to dehydrated hAM. ${ }^{8,9}$ Cryopreservation protocols, however, are not standardized, which results in high variability in cellular integrity. For example, reported cell viability in hAM processed by different cryopreservation protocols varies from the complete loss of viable cells to the retention of $50 \%$ viable cells. ${ }^{10-12}$ Because devitalized hAM (devhAM) exhibits therapeutic effects, it has been suggested that the preservation of viable cells in hAM provides no additional benefits. ${ }^{12}$ This conclusion led to the development of cryopreservation methods that include hAM devitalization steps. One of them, known as the CryoTek ${ }^{\mathrm{TM}}$ method, includes a freezing step before cryopreservation, resulting in devitalized tissue. ${ }^{13}$ However, others have demonstrated that the combination of exogenous cells and acellular hAM resulted in faster wound closure in comparison to acellular hAM alone. ${ }^{14}$ Therefore, we hypothesized that the functionality of intact, cryopreserved hAM (int-hAM), containing viable endogenous cells, would be better than that of dev-hAM. This study investigates the anti-inflammatory activities of int-hAM and dev-hAM.

\section{CLINICAL PROBLEM ADDRESSED}

There is considerable variability in the tissue composition of hAM processed by different preservation methods. The relationship between tissue preservation methods and the anti-inflammatory activity of hAM has not been established. Our data illustrate that the preservation of viable endogenous cells enhances the anti-inflammatory activity of cryopreserved hAM. The findings in this study contribute to our understanding of the role of hAM components in its functionality.

\section{MATERIALS AND METHODS}

\section{Tissue procurement and ethics statement}

Human term placentas were provided by The National Disease Research Interchange (Philadelphia, PA) and Cord Blood America, Inc. (Las Vegas, NV) from eligible donors after obtaining written, informed consent.

\section{Placental tissue processing}

Placental tissues were aseptically processed in a biological safety cabinet within $36 \mathrm{~h}$ after collection. The hAM was separated from the umbilical cord and from the chorion by blunt dissection. Residual blood was removed, and the hAM was incubated in Dulbecco's modified Eagle's medium (DMEM) (GE Healthcare Life Sciences, Piscataway, $\mathrm{NJ})$ containing an antibiotic cocktail of gentamicin (Fresenius Kabi USA, Lake Zurich, IL), vancomycin (Hospira, San Jose, CA), and Amphotericin B (Sigma-Aldrich, St. Louis, MO) for 18-48 h at $37^{\circ} \mathrm{C}$ and $5 \% \mathrm{CO}_{2}$ in a humidified atmosphere. Subsequently, residual antibiotics were removed by washing with Dulbecco's phosphate-buffered saline (DPBS) (Life Technologies, Carlsbad, CA), and the hAM was cut into $25 \mathrm{~cm}^{2}$ and $4 \mathrm{~cm}^{2}$ pieces. Unless specified, $25 \mathrm{~cm}^{2}$ pieces were used in the following experiments.

Cryopreservation of hAM was performed by freezing hAM in a dimethyl sulfoxide (DMSO) (Mylan, Inc., Canonsburg, PA) containing cryoprotectant solution at a controlled cooling rate, according to a proprietary process developed by Osiris Therapeutics, Inc. (Columbia, MD). Tissues that were prepared solely by cryopreservation will be referred to as int-hAM in the study. Tissues prepared with cryopreservation and an additional freezing step that resulted in devitalization will be referred to as dev-hAM. Int-hAM and dev-hAM were stored at $-80^{\circ} \mathrm{C}$ for up to 3 months before their use in the experiments. Both int-hAM and dev-hAM were thawed in a room temperature water bath and rinsed with room temperature saline or DPBS before use. Placentas from multiple donors were used in this study.

\section{Histological evaluation}

Fresh hAM, int-hAM, and dev-hAM samples were fixed in $10 \%$ formalin for $24 \mathrm{~h}$ and then transferred to ethanol. Samples were embedded in paraffin, sectioned into $5 \mu \mathrm{m}$-thick cross-sections, and stained with 
hematoxylin and eosin (H\&E) according to standard protocols at Histoserv, Inc. (Germantown, MD).

\section{Immunohistological staining}

After being deparaffinized, rinsed, and blocked with $4 \%$ bovine serum, fresh hAM, int-hAM, and dev-hAM were incubated with primary antibodies against collagen IV (1:500) (Abcam, Cambridge, MA) at room temperature overnight, followed by secondary antibodies (1:500) and streptavidin-horseradish peroxidase (1:400) (Vector Laboratories, Burlingame, $\mathrm{CA})$, respectively. Stained collagen IV was visualized using 3,3'-diaminobenzidine. Sections were counterstained with hematoxylin (blue nuclei stain). Tissue sections incubated in the absence of primary antibodies served as a negative control. Immunohistological staining was performed by Histoserv, Inc.

\section{Assessment of placental tissue cell viability}

A quantitative assessment of the cell viability of tissue samples was performed using the trypan blue exclusion method. Fresh hAM, int-hAM, and dev-hAM were digested in $0.75 \mathrm{mg} / \mathrm{mL}$ Collagenase Type II solution (Worthington Biochemical Corporation, Lakewood, NJ) in DMEM and incubated with mild agitation at $37^{\circ} \mathrm{C}$ for $30 \mathrm{~min}$. Subsequently, collagenase was removed by centrifugation, and $0.05 \%$ trypsin-ethylenediaminetetraacetic acid (EDTA) solution (Life Technologies) was added to the remaining pellets for further digestion. After 5$10 \mathrm{~min}$, cells released from the tissue were washed with DMEM and pelleted by centrifugation. Cells were counted using a hemocytometer under an inverted microscope (Eclipse TE300; Nikon, Tokyo, Japan), and viable cells were identified by exclusion of the trypan blue dye (Sigma-Aldrich).

For a qualitative assessment of cell viability, the LIVE/DEAD ${ }^{\circledR}$ viability/cytotoxicity kit (Life Technologies) was used according to the manufacturer's instructions. Briefly, tissue samples were incubated in a 1:1,000 dilution of both components of the kit for $30 \mathrm{~min}$. Samples were then visualized under a fluorescent microscope (Eclipse TE300; Nikon). Viable, intact cells within the tissue pieces were identified by green fluorescent calcein AM, whereas dead or damaged cells were labeled with red fluorescent ethidium homodimer-1. Photographs were taken for both green and red channels, which were then merged using ImageJ, a Java ${ }^{\mathrm{TM}}$ based image processing program provided by the National Institute of Health (Bethesda, MD).

To evaluate cell viability after culture for an extended period, int-hAM samples were incubated in DMEM supplemented with $10 \%$ fetal bovine serum (FBS) (Life Technologies) and $2 \%$ antibioticantimycotic (Life Technologies) for 7 days. The viability of int-hAM samples was then assessed qualitatively using the LIVE/DEAD viability/ cytotoxicity kit as described earlier.

\section{Evaluation of cytokine and growth factor profile in int-hAM and dev-hAM}

A protein profile of int-hAM and dev-hAM tissue extracts was investigated using a multiplex chemiluminescent array. For tissue lysates, inthAM and dev-hAM were snap-frozen in liquid nitrogen followed by pulverization using a mortar and pestle. Crushed tissue was transferred to $500 \mu \mathrm{L}$ of cell lysis buffer (Cell Signaling Technology, Danvers, MA) with protease inhibitor (Roche, Basel, Switzerland) and incubated on ice for $30 \mathrm{~min}$ with frequent vortexing. Tissue lysates were then centrifuged at $16,000 \mathrm{~g}$ for $10 \mathrm{~min}$. Supernatants were collected and sent for protein array analysis on dry ice. Analysis was performed at Aushon Biosystems (Billerica, MA). All samples were tested at three dilutions for 36 proteins, including the following proteases, protease inhibitors, angiogenic factors, growth factors, and cytokines known to be important for wound healing: matrix metalloproteinase (MMP)-1, MMP-2, MMP-3, MMP7, MMP-8, MMP-9, MMP-10, MMP-13, tissue inhibitor of metalloproteinase (TIMP)-1, TIMP-2, angiotensin-2, basic fibroblast growth factor (bFGF), epidermal growth factor (EGF), heparin-bound EGF, keratinocyte growth factor, platelet-derived growth factor (PDGF)-AA, PDGF-AB, PDGF-BB, vascular endothelial growth factor (VEGF)-A, VEGF-C, VEGF-D, neutrophil gelatinase-associated lipocalin (N-Gal), hepatocyte growth factor (HGF), placental growth factor, pigment epitheliumderived factor, thrombopoietin, $\alpha$-2-macroglobulin, adiponectin, transforming growth factor (TGF)- $\alpha$, granulocyte colony-stimulating factor, interleukin1 receptor antagonist (IL-1RA), leukemia inhibitory factor, stromal-derived factor- $1 \beta$, insulin-like growth factor binding protein (IGFBP)-1, IGFBP-2, and IGFBP-3. ${ }^{15}$

\section{Effect of int-hAM and dev-hAM on cytokine secretion by activated human peripheral blood mononuclear cells}

The anti-inflammatory activity of int-hAM and dev-hAM was determined by measuring the change in the secretion of pro-inflammatory cytokines tumor necrosis factor- $\alpha$ (TNF- $\alpha)$ and interleukin- $1 \alpha$ (IL-1 $\alpha)$ and anti-inflammatory cytokine interleukin-10 (IL-10) by human peripheral blood mononuclear cells (hPBMCs) (SeraCare Life Sciences, Milford, MA).

To determine the effect of hAM on the secretion of TNF- $\alpha$ and IL- $1 \alpha, 1.0 \times 10^{6} \mathrm{hPBMCs}$ were activated with anti-cluster of differentiation (CD)3 
and anti-CD28 monoclonal antibodies (BD Biosciences, Franklin Lakes, NJ) at $4 \mu \mathrm{g} / \mathrm{mL}$. Activated hPBMCs were cultured with int-hAM or dev-hAM for $48 \mathrm{~h}$ at $37^{\circ} \mathrm{C}$ and $5 \% \mathrm{CO}_{2}$ in a humidified atmosphere. Positive and negative controls were hPBMCs alone with and without anti-CD3 and anti-CD28 antibodies, respectively. After $48 \mathrm{~h}$ of incubation, supernatants were collected by centrifuging samples at $300 \mathrm{~g}$ for $5 \mathrm{~min}$ and stored at $-80^{\circ} \mathrm{C}$ until testing by DuoSet ${ }^{\circledR}$ enzyme-linked immunosorbent assay (ELISA) (R\&D Systems, Minneapolis, MN).

To determine the effect of hAM on the secretion of IL-10, $1.0 \times 10^{6} \mathrm{hPBMCs}$ were prestimulated with $100 \mathrm{ng} / \mathrm{mL}$ lipopolysaccharide (LPS) (SigmaAldrich) for $4 \mathrm{~h}$ before the addition of $12 \mathrm{~cm}^{2}$ inthAM or dev-hAM, which were pretreated with $10 \mathrm{ng} / \mathrm{mL}$ TNF- $\alpha$ for $18 \mathrm{~h}$. LPS-activated hPBMCs and hAM were co-cultured for an additional $24 \mathrm{~h}$ at $37^{\circ} \mathrm{C}$ and $5 \% \mathrm{CO}_{2}$ in a humidified atmosphere. Positive and negative controls were hPBMCs alone with and without LPS stimulation, respectively. After $24 \mathrm{~h}$ of incubation, conditioned medium was collected by centrifuging samples at $300 \mathrm{~g}$ for $10 \mathrm{~min}$ and stored at $-80^{\circ} \mathrm{C}$ until further testing by DuoSet ELISA (R\&D Systems). The cell viability of hPBMCs in each group was evaluated using the trypan blue exclusion method immediately after the collection of conditioned medium.

\section{Tumor necrosis factor- $\alpha$-induced release of prostaglandin E2}

Int-hAM and dev-hAM tissues $\left(4 \mathrm{~cm}^{2}\right.$ each) were incubated in $1 \mathrm{~mL}$ of low serum culture medium (1\% FBS in DMEM) in the absence or presence of $50 \mathrm{ng} / \mathrm{mL}$ TNF- $\alpha$ (R\&D Systems) for $20 \mathrm{~h}$ at $37^{\circ} \mathrm{C}$ and $5 \% \mathrm{CO}_{2}$ in a humidified atmosphere. Samples without the addition of TNF- $\alpha$ were considered baseline controls. The supernatants were collected, centrifuged at $300 \mathrm{~g}$ for $10 \mathrm{~min}$, stored at $-80^{\circ} \mathrm{C}$, and thawed just before testing. The levels of prostaglandin E2 (PGE2) were quantified using the PGE2 Express ELISA kit (Cayman Chemical, Ann Arbor, MI) according to the manufacturer's protocol. The relative \% change was calculated using the following formula: [( $\mathrm{PGE} 2$ release in the presence of TNF- $\alpha)-($ PGE2 release of baseline control))/PGE2 release at baseline control]*100.

\section{Effect of int-hAM and dev-hAM on collagenase activity}

An azocoll (azo-dye impregnated collagen) protease activity assay was performed using a modified protocol described by Jiang et al. ${ }^{16}$ Azocoll (SigmaAldrich) was washed with DPBS for $6 \mathrm{~h}$ to remove any degraded peptides. The washed azocoll was re- suspended in fresh DPBS at a final concentration of $1.5 \mathrm{mg} / \mathrm{mL}$. Collagenase Type IV (Life Technologies) at a final concentration of $0.1 \%$ (weight/volume), azocoll at a final concentration of $0.5 \mathrm{mg} / \mathrm{mL}$, and int-hAM or dev-hAM were incubated for $5 \mathrm{~h}$ on an end-to-end rotator at $37^{\circ} \mathrm{C}$. Collagenase and azocoll incubated under the same conditions without the tissue samples served as a positive control. Azocoll alone served as a negative control. The enzymatic reaction was stopped by centrifugation of the samples at $10,000 \mathrm{~g}$ for $8 \mathrm{~min}$. The absorbance of the samples was measured at $550 \mathrm{~nm}$ using a spectrophotometer (SpectraMax; Molecular Devices, Sunnyvale, CA).

\section{Effect of int-hAM and dev-hAM on neutrophil elastase activity}

To evaluate the inhibitory effect of hAM on elastase, int-hAM and dev-hAM were pretreated with $100 \mathrm{ng}$ of human neutrophil elastase (SigmaAldrich) in DMEM with $1 \% \mathrm{FBS}$ for $24 \mathrm{~h}$ at $37^{\circ} \mathrm{C}$ and $5 \% \mathrm{CO}_{2}$ in a humidified atmosphere. The pretreated tissue samples and $100 \mathrm{ng}$ of fresh neutrophil elastase were incubated for $4 \mathrm{~h}$ at $37^{\circ} \mathrm{C}$ in a final volume of $500 \mu \mathrm{L}$ of $0.1 \mathrm{M}$ HEPES [4-(2-hydroxyethyl)1-piperazineethanesulfonic acid] buffer, $\mathrm{pH} 7.4$ (Sigma-Aldrich), containing 0.5 M sodium chloride (Sigma-Aldrich), 10\% DMSO, and $1 \mathrm{mM}$ elastase substrate (N-methoxysuccinyl-Ala-Ala-Pro-Val-p-nitroanilide) (Sigma-Aldrich). Elastase and elastase substrate incubated under the same conditions without hAM served as a positive control. Elastase substrate alone served as a negative control. The enzymatic reaction was stopped by centrifugation of the samples at $10,000 \mathrm{~g}$ for $8 \mathrm{~min}$. The absorbance of the supernatants was measured at $405 \mathrm{~nm}$ using a spectrophotometer (SpectraMax; Molecular Devices).

\section{Statistical analysis}

Data are presented as mean \pm standard (SD) deviation for one representative experiment containing three replicates. Unless otherwise specified, the $\%$ change of int-hAM or dev-hAM from positive control was calculated using the following formula: [((value for positive control)-(value for int-hAM or dev-hAM))/value for positive control]*100. The magnitude of effect of int-hAM relative to dev-hAM, expressed as \% of dev-hAM, was calculated using the following formula: [ $\%$ change of int-hAM $) /(\%$ change of dev-hAM)]*100. In instances where the \% change was negative, the magnitude of the effect of int-hAM relative to devhAM was calculated using the following formula: ([(\% change of int-hAM) - (\% change of dev-hAM)]/ [absolute value of (\% change of dev-hAM)] $* 100$. Student's $t$-test was used for statistical analysis, and $p<0.05$ was considered significant. 

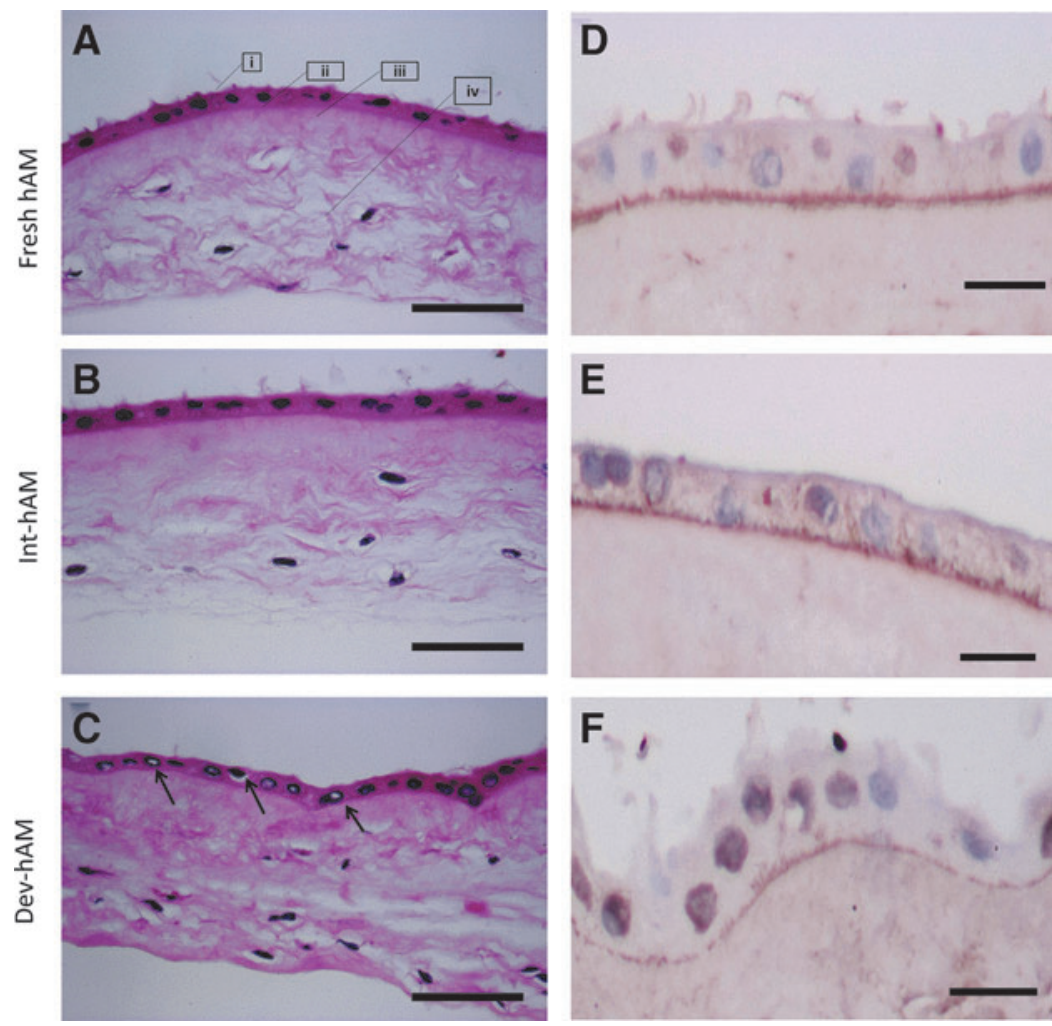

Figure 1. Hematoxylin and eosin (H\&E) and collagen IV (Col IV) staining of human amniotic membranes (hAMs). H\&E staining of (A) fresh hAM, (B) viable intact cryopreserved hAM (int-hAM), and (C) devitalized cryopreserved hAM (dev-hAM). Layers of hAM are shown in (A): (i) epithelial layer, (ii) basement membrane, (iii) compact layer, and (iv) stromal layer. Intracytoplasmic vacuoles in the epithelial cells (arrows) are shown in (C). Brown-colored stained Col IV in the basement membrane of (D) fresh-hAM, (E) int-hAM, and (F) dev-hAM. Scale bars: $50 \mu \mathrm{m}$ (H\&E) and $20 \mu \mathrm{m}$ (Col IV).

\section{RESULTS}

\section{Evaluation of structural and cellular integrity of int-hAM and dev-hAM}

To investigate the structural integrity of int-hAM and dev-hAM, a histological analysis was performed, which included H\&E and immunohistochemical col- lagen IV staining. Fresh hAM served as a reference control. Fresh hAM contains an epithelial layer, basement membrane, compact layer, and stromal layer (Fig. 1A). ${ }^{17}$ When compared with fresh hAM, int-hAM showed no changes in the tissue architecture (Fig. 1B). Dev-hAM, prepared by freezing and cryo-

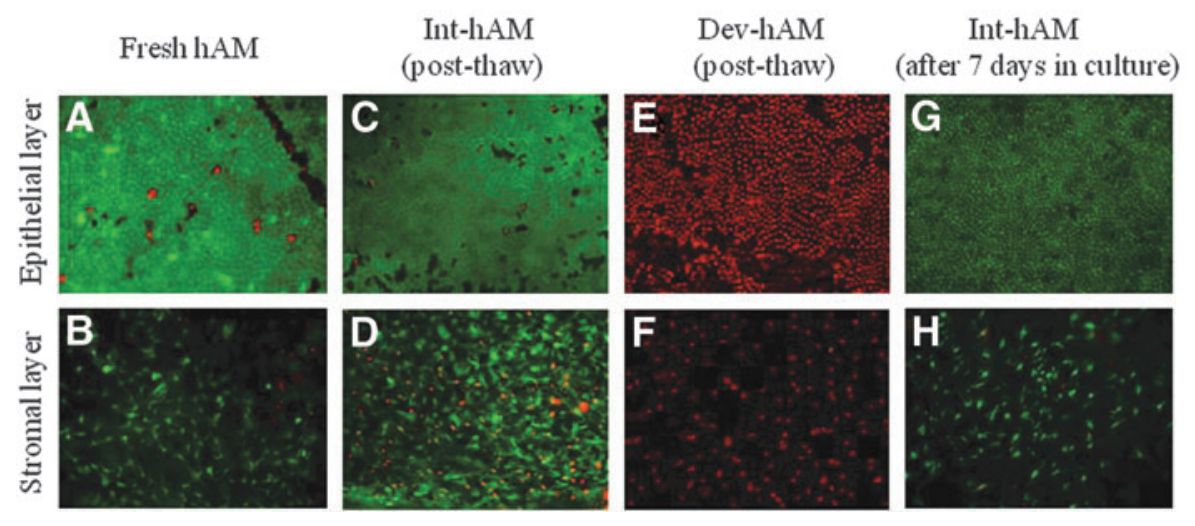

Figure 2. Live and dead endogenous cell staining of human amniotic membrane (hAM). Live and dead cells in epithelial and stromal layers of fresh hAM (A, B), viable intact cryopreserved hAM (int-hAM) post-thaw (C, D), and devitalized cryopreserved hAM (dev-hAM) post-thaw (E, F) were visualized microscopically using the LIVE/DEAD ${ }^{\circledR}$ viability/cytotoxicity kit. Live cells stained green with calcein AM. Dead cells stained red with ethidium homodimer-1. Live and dead cells in int-hAM after 7 days in culture are shown in (G, H). All images were taken at $10 \times$ magnification. 
preservation, showed the presence of intracytoplasmic vacuoles in the epithelial cells (Fig. 1C).

Collagen IV is an ECM protein found primarily in the basement membrane of hAM. ${ }^{18}$ Immunohistochemical staining for collagen IV revealed that fresh hAM contains a basement membrane that is rich in collagen IV as depicted by the brown staining underneath the epithelial layer (Fig. 1D). This expression of collagen IV was maintained after cryopreservation in int-hAM (Fig. 1E). Minor degradation in collagen IV was observed in dev-hAM shown as thinner and weaker brown staining in Fig. $1 \mathrm{~F}$.

In Fig. 1, endogenous cells appeared to be the same in both int-hAM and dev-hAM, as they both contain blue nuclear staining and pink cytoplasmic staining. However, histological staining does not distinguish viable from dead cells. Therefore, we examined the viability of endogenous cells in inthAM and dev-hAM post-thaw using the LIVE/ DEAD viability/cytotoxicity assay. Fresh hAM has an epithelial layer containing a continuous sheet of cuboidal, neonatal epithelial cells and a stromal layer containing neonatal fibroblasts and mesenchymal stem cells. ${ }^{19}$ The viability of the endogenous cells was maintained in int-hAM post-thaw similar to fresh hAM (Fig. 2A-D). In comparison, dev-hAM contained only dead cells (Fig. 2E, F). Furthermore, a trypan blue exclusion assay was performed to quantify cell viability within fresh hAM and int-hAM. Our data showed that on average, $84 \% \pm 3 \%$ and $82 \% \pm 6 \%$ of viable cells were detected in fresh hAM and int-hAM, respectively. To examine the persistence of viable cells in inthAM over 7 days in culture, a LIVE/DEAD viability/cytotoxicity assay was again performed. Figure $2 \mathrm{G}$ and $\mathrm{H}$ demonstrate that the majority of cells in both the epithelial and stromal layers of int-hAM remained viable over time (comparable to day 0 ).
The presence of growth factors and cytokines in int-hAM and dev-hAM was analyzed using a multiplex protein array. Thirty-six proteins (listed in the Materials and Methods section), which are important for wound healing, were selected for testing. We found that both int-hAM and dev-hAM contained all of these growth factors and cytokines. The ranges for several selected wound-healing factors among the 36 tested proteins in int-hAM and dev-hAM were as follows: $77-157 \mathrm{pg} / \mathrm{cm}^{2}$ for TGF- $\beta 1 ; 246-941 \mathrm{pg} / \mathrm{cm}^{2}$ for HGF; $243-2,019 \mathrm{pg} /$ $\mathrm{cm}^{2}$ for IL-1RA; $3.0-9.4 \mathrm{pg} / \mathrm{cm}^{2}$ for VEGF; 1.0 $7.5 \mathrm{pg} / \mathrm{cm}^{2}$ for PDGF; $7.5-51 \mathrm{pg} / \mathrm{cm}^{2}$ for $\mathrm{bFGF}$; and $386-431 \mathrm{pg} / \mathrm{cm}^{2}$ for N-Gal.

To determine the effect of structural and cellular preservation of hAM on its anti-inflammatory activity, int-hAM and dev-hAM were compared in the following experiments.

\section{Inhibition of TNF- $\alpha$ and IL-1 $\alpha$ secretion by activated hPBMCs}

We used hPBMCs as a source of inflammatory cells. Polyclonal stimuli, such as anti-CD3 and anti-CD28 antibodies, trigger secretion of inflammatory cytokines from hPBMCs. ${ }^{20}$ Without stimulation, the release of TNF- $\alpha$ and IL- $1 \alpha$ by hPBMCs was below detectable levels. When stimulated by anti-CD3 and anti-CD28, hPBMCs released $1,852 \pm 39 \mathrm{pg} / \mathrm{mL}$ TNF- $\alpha$ and $4,499 \pm 221 \mathrm{pg} / \mathrm{mL}$ IL-1 $\alpha$, respectively (Fig. 3A, B). When int-hAM or dev-hAM were added to activated hPBMCs, inthAM showed a statistically significant downregulation of inflammatory cytokines as compared with dev-hAM for both TNF- $\alpha(<15.6 \mathrm{pg} / \mathrm{mL}$ [lowest quantifiable value was assigned] vs. $1,171 \pm 12 \mathrm{pg} /$ $\mathrm{mL}$, respectively) and IL- $1 \alpha(24 \pm 29 \mathrm{pg} / \mathrm{mL}$ vs. $1,966 \pm 505 \mathrm{pg} / \mathrm{mL}$, respectively) (Fig. 3A, B). Neither int-hAM nor dev-hAM secreted detectable
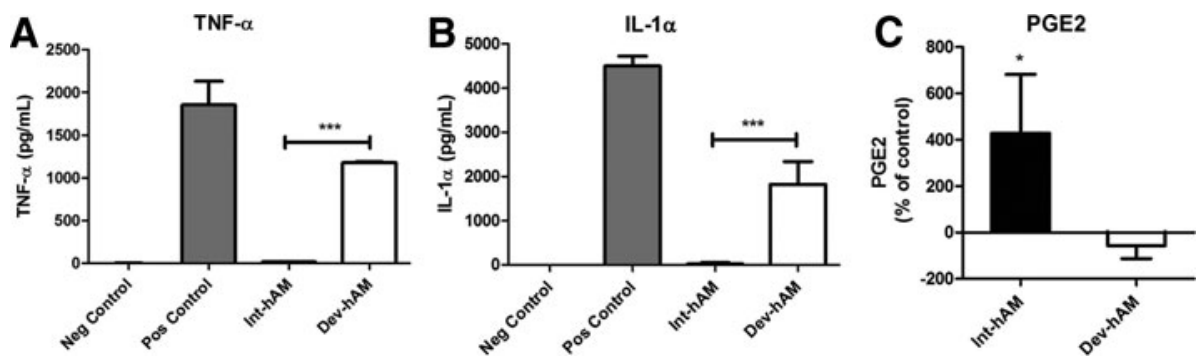

Figure 3. Effects of human amniotic membrane (hAM) on human peripheral blood mononuclear cell (hPBMC)-induced pro-inflammatory factor release. Inhibition of activated hPBMC-derived tumor necrosis factor- $\alpha$ (TNF- $\alpha)$ (A) and interleukin-1 $\alpha($ IL-1 $\alpha)$ (B) in the presence of viable intact cryopreserved hAM (int-hAM) and devitalized cryopreserved hAM (dev-hAM). Negative (Neg) and positive (Pos) controls were unstimulated and anti-cluster of differentiation (CD)3 and anti-CD28-activated hPBMCs, respectively. Levels of prostaglandin E2 (PGE2) released from int-hAM and dev-hAM following TNF- $\alpha$ stimulation are shown in (C). PGE2 levels were calculated as $\%$ of PGE2 released in cultures without TNF- $\alpha$ ( $\%$ of control). Data shown represent the mean \pm SD of one experiment performed in triplicate. Student's $t$-test was used for statistical analysis. ${ }^{*} p<0.05$ and ${ }^{* * *} p<0.001$. 
amounts of TNF- $\alpha$ and IL- $1 \alpha$ with or without antiCD3 and anti-CD28 antibodies (data not shown). The viability of hPBMCs was not affected by hAM tissues. In all groups, hPBMCs were $>75 \%$ viable, and there was no difference between the controls (hPBMCs alone) and co-cultures (hPBMCs with inthAM and with dev-hAM) (data not shown).

\section{Increase in PGE2 release by int-hAM in response to TNF- $\alpha$}

We hypothesized that the downregulation of the pro-inflammatory cytokines observed in the previous experiment might be mediated by PGE2, as PGE2 is known to inhibit immune cell proliferation and pro-inflammatory cytokine production. ${ }^{21}$ It has been shown that not only does hAM matrix contain PGE2, but also hAM cells can produce additional PGE2 upon stimulation. ${ }^{22,23}$ We exposed hAM to TNF- $\alpha$ and measured the level of PGE2 in the supernatant. The baseline levels of PGE2 from the two groups were not significantly different (an average of $2,872 \mathrm{pg} / \mathrm{cm}^{2}$ ). This level is consistent with a previous report. ${ }^{23}$ A $428 \%$ increase in PGE2 from baseline was observed for int-hAM, which contrasted with the 56\% decrease in PGE2 for devhAM (Fig. 3C).

\section{Increase in IL-10 release in a co-culture of LPS-stimulated hPBMCs and int-hAM}

Activation of $\mathrm{hPBMCs}$ by LPS was used to detect the production of anti-inflammatory cytokine IL10. When LPS-activated hPBMCs were incubated with TNF- $\alpha$ pretreated tissues, we observed an increase in IL-10 release $(255 \pm 81 \mathrm{pg} / \mathrm{mL})$ and a decrease in IL-10 release $(86 \pm 20 \mathrm{pg} / \mathrm{mL})$ relative to the positive control $(174 \pm 27 \mathrm{pg} / \mathrm{mL})$ for int-hAM and dev-hAM, respectively (Fig. 4).

\section{Int-hAM protects substrates from protease-mediated degradation}

In addition to high levels of inflammatory cytokines, another hallmark of chronic wounds is high levels of proteases, such as MMPs and elastase, that degrade ECM and growth factors. ${ }^{24}$ First, we evaluated the ability of int-hAM and dev-hAM to protect collagen from degradation by collagenase using the azocoll assay. Int-hAM showed significantly greater inhibition of collagenase compared to dev-hAM $(0.09 \pm 0.03$ vs. $0.3 \pm 0.1$ absorbance, Fig. 5A). This corresponds to $74 \%$ versus $7 \%$ inhibition of collagenase with int-hAM and dev-hAM, respectively.

Similarly, we evaluated the effect of int-hAM and dev-hAM on neutrophil elastase activity. Inhibition of elastase activity was assayed using an elastase

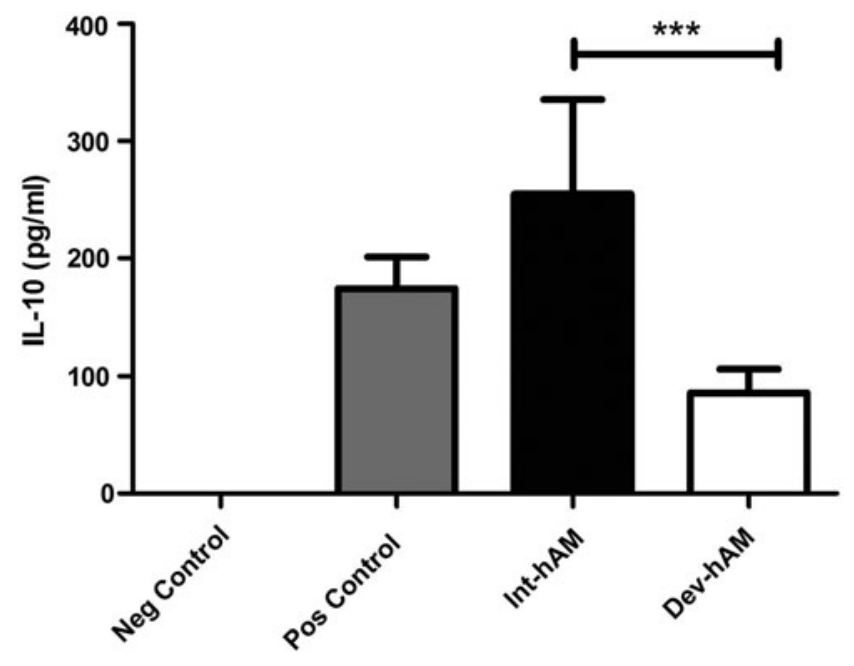

Figure 4. Effects of human amniotic membrane (hAM) on human peripheral blood mononuclear cell (hPBMC)-induced anti-inflammatory factor interleukin-10 (IL-10) release. IL-10 levels in conditioned medium were measured after $24 \mathrm{~h}$ co-cultures of lipopolysaccharide (LPS)-stimulated hPBMCs with viable intact cryopreserved hAM (int-hAM) and devitalized cryopreserved hAM (dev-hAM). Negative (Neg) and positive (Pos) controls were unstimulated and LPS-activated hPBMCs, respectively. Data shown represent the mean $\pm S D$ of one experiment performed in triplicate. Student's $t$-test was used for statistical analysis. ${ }^{* * *} p<0.001$.

substrate. Both int-hAM and dev-hAM suppressed the substrate cleavage by neutrophil elastase. However, int-hAM-mediated inhibition of elastase activity was significantly stronger than dev-hAM $(0.17 \pm 0.04$ vs. $0.62 \pm 0.09$ absorbance, Fig. $5 B)$. This corresponds to $94 \%$ versus $78 \%$ inhibition of elastase with int-hAM and dev-hAM, respectively.

\section{DISCUSSION}

Different preservation methods have been developed to overcome the limitations of fresh hAM and to ensure an on-demand supply of hAM for clinical use. However, the majority of these methods result in the destruction of endogenous cells and in varying degrees of ECM damage, which may impact the functionality of hAM and thereby its clinical benefits for wound treatment. Here, we introduced a cryopreservation method that retains viable hAM cells in their native state. We compared the tissue composition and anti-inflammatory activity between int-hAM and dev-hAM.

Int-hAM was prepared with a DMSO-based cryopreservation solution using a proprietary process. Dev-hAM was prepared by including an extra freezethaw step that destroyed viable endogenous cells. Our histological analysis confirmed that int-hAM maintains the matrix integrity of fresh hAM (Fig. 1A-D). Minor matrix degeneration was observed in 
A

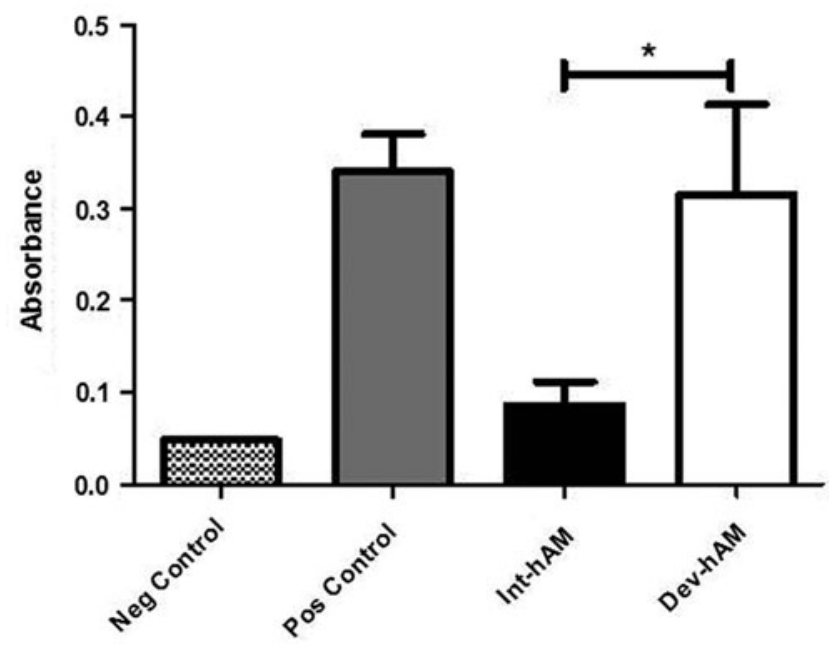

B

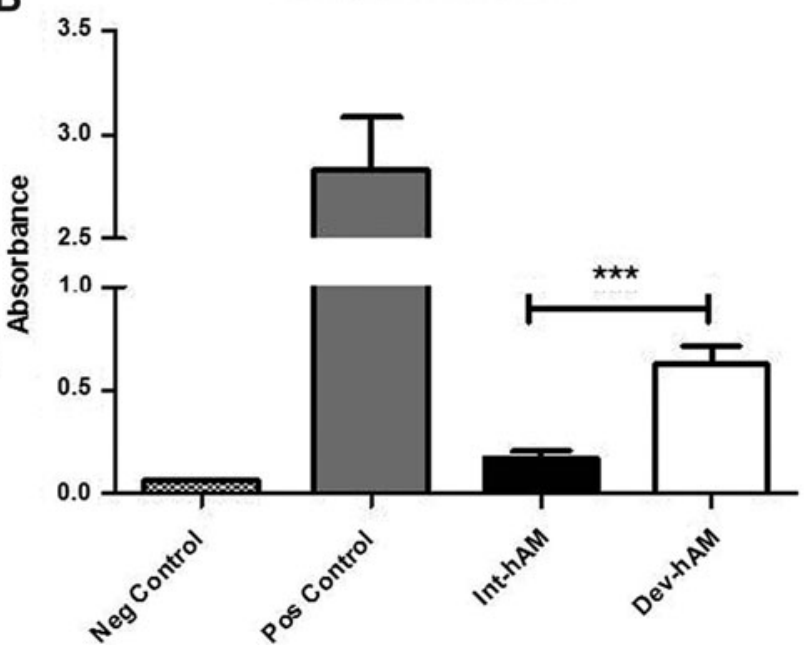

Figure 5. Effects of human amniotic membrane (hAM) on protease activities. Substrate cleavage by (A) Collagenase Type IV and (B) neutrophil elastase in the presence of viable intact cryopreserved hAM (int-hAM) and devitalized cryopreserved hAM (dev-hAM) was measured spectrophotometrically and presented in absorbance. Proteases hydrolyze substrates and yield soluble colored peptides in proportion to enzyme activity. Collagenase Type IV activity was assayed using azocoll as a substrate. Elastase activity was assayed using methoxysuccinyl-ala-ala-pro-val-p-nitroanilide as a substrate. Negative (Neg) and positive (Pos) controls were substrates alone and substrates incubated with proteases, respectively. Data shown represent the mean $\pm S D$ of one experiment performed in triplicate. Student's $t$-test was used for statistical analysis. ${ }^{*} p<0.05$ and ${ }^{* *} p<0.001$.

dev-hAM, but the architecture of the matrix structure was largely preserved (Fig. 1C, F). This finding is in agreement with previous reports. ${ }^{9,25}$ In addition, the presence of growth factors and cytokines that were important for wound healing in both int-hAM and dev-hAM tissue was confirmed using a protein array.

A comparison of the cell viability in fresh hAM and int-hAM revealed that on average, the percentage of viable cells was $>80 \%$ for both groups with no sig- nificant difference between the two. Moreover, cells in int-hAM remained viable in culture for at least 1 week, comparable to day 0 (Fig. $2 \mathrm{G}, \mathrm{H}$ ). To date, $50 \%$ or less cell viability of cryopreserved hAM postthaw has been reported, and cells failed to survive after 18 months of storage at $-80^{\circ} \mathrm{C}^{10,11}$ The cryopreservation method reported in this study, however, maintains $70 \%$ or greater cell viability in int-hAM even after 24 months of storage at $-80^{\circ} \mathrm{C}$ (data not shown).

For the treatment of wounds, hAM's antiinflammatory activity is especially important. Therefore, we evaluated the anti-inflammatory activity of int-hAM and dev-hAM. Int-hAM inhibited the secretion of pro-inflammatory cytokines TNF- $\alpha$ and IL- $1 \alpha$ almost completely. In comparison, dev-hAM showed significantly weaker inhibition: an average of $40 \%$ for TNF- $\alpha$ and $56 \%$ for IL- $1 \alpha$. Thus, int-hAM shows $148 \%$ and $77 \%$ greater inhibition of TNF- $\alpha$ and IL- $1 \alpha$ compared with dev-hAM, respectively (Fig. 6).

The anti-inflammatory properties of hAM are complex and are mediated by different factors and pathways. Previously, several different mechanisms had been proposed: high-molecular-weight hyaluronic acid (HA), a complex of HA and a heavy chain of inter- $\alpha$-inhibitor, PGE2, IL-10, and IL1RA. ${ }^{22}$ Other factors that may also be involved in hAM anti-inflammatory activity include TGF- $\beta$, $\mathrm{HGF}$, and indoleamine 2, 3-dioxygenase. ${ }^{22}$

Among these molecules, PGE2 is known to be present in the hAM matrix, and it can be secreted

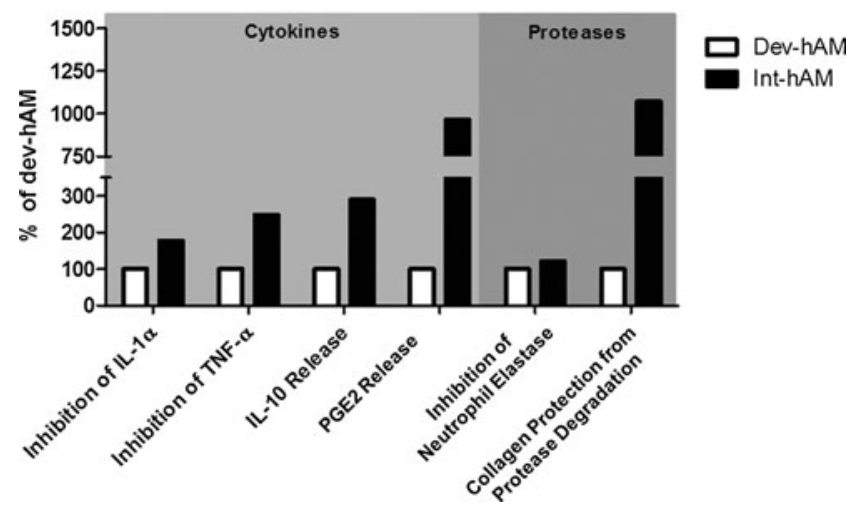

Figure 6. Summary of viable intact cryopreserved human amniotic membrane (int-hAM) and devitalized cryopreserved human amniotic membrane (dev-hAM) properties. The magnitude of effect between int-hAM and dev-hAM was calculated using the following formula: ( $\%$ change of int-hAM)/(\% change of dev-hAM) $)^{*} 100$. In instances where the $\%$ change was negative, magnitude of effect of int-hAM relative to dev-hAM, expressed as $\%$ of dev-hAM, was calculated using the following formula: ([l\% change of int-hAM)- $(\%$ change of dev-hAM $)] /[$ absolute value of $(\%$ change of dev-hAM)] $)^{*} 100$. 
by hAM's epithelial and stromal cells. ${ }^{22,26}$ When PGE2 synthesis is blocked, the anti-inflammatory effects of hAM-derived cells are diminished. ${ }^{27}$ In our study, a $428 \%$ increase in PGE2 release from inthAM was observed after stimulation with TNF- $\alpha$ in comparison to a decreased release in PGE2 from devhAM (Fig. 3C). This suggests that PGE2 may represent one mechanism by which int-hAM inhibits secretion of TNF- $\alpha$ and IL- $1 \alpha$ by activated hPBMCs.

In addition to the inhibition of pro-inflammatory cytokines, int-hAM showed $189 \%$ higher antiinflammatory cytokine IL-10 expression relative to dev-hAM when cultured with LPS-activated hPBMCs (Fig. 6). IL-10 has been found to be present in hAM, so the increase in IL-10 could be due to its release from int-hAM cells. ${ }^{5,22,28}$ On the other hand, activated hPBMCs alone secreted IL-10 (Fig. 4). The observed effect of int-hAM could be mediated by an indirect mechanism in which inthAM upregulates IL-10 release by hPBMCs. IL-10 release represents another mechanism by which int-hAM inhibits inflammation.

The prolonged inflammatory reaction in chronic wounds leads to a protease imbalance that is believed to be a crucial factor in the development of chronic wounds and is associated with delayed healing. ${ }^{24}$ Elevated quantities of proteolytic enzymes, such as elastase and MMPs, are released within the chronic wound, in part, as a result of excessive infiltration and reduced clearance of neutrophils. ${ }^{24,29}$ In comparison to acute wounds, MMP activity in chronic wound fluid is 30 times higher. ${ }^{29}$ High levels of proteases lead to the rapid degradation of growth factors and ECM proteins such as collagen. ${ }^{24,30-32}$ The breakdown products of protease degradation further promote an inflammatory reaction, and thus, the inflammatory phase is transitioned from a self-limiting to a self-sustaining process. ${ }^{33}$ Protection of the ECM and growth factors in the chronic wound environment via the modulation of protease activity is essential for shutting down inflammation and transitioning the wound into the next stage of wound healing.

The effect of int-hAM on inhibition of protease activity was examined in this study. Int-hAM showed a $908 \%$ increase in its ability to protect collagen from degradation and a $20 \%$ increase in elastase inhibition compared with dev-hAM (Fig. 6). This inhibition may be mediated via protease inhibitors in hAM: TIMP-1, thrombospondin-1, and plasminogen activator inhibitior- $1 .{ }^{34,35}$ In addition, hAM is known to produce elafin and secretory leukocyte protease inhibitor, which inhibit elastase activity and are strongly associated with the woundhealing process. ${ }^{36-38}$ The superior inhibition of col- lagenase by int-hAM as compared with dev-hAM may be mediated by multiple mechanisms. One mechanism could be a greater amount of protease inhibitors in the tissue and/or due to the release of inhibitors by the viable cells in response to the high protease level. Another mechanism could be the presence of a higher amount of intact collagen in int$\mathrm{hAM}$ than in dev-hAM. Intact collagen in int-hAM could potentially mediate int-hAM's inhibitory effect by competing with the azocoll substrate in the assay. However, histological evaluation does not show significant matrix degradation in dev-hAM (Fig. 1C). Therefore, it is unlikely that substrate competition is the main mechanism for collagenase inhibition. Understanding the individual contribution of the earlier mentioned molecules and mechanisms and how cryopreservation of hAM affects them are subjects for further evaluation.

In summary, obtained data demonstrate that int-hAM has significantly higher anti-inflammatory activity in comparison to dev-hAM (Fig. 6). These results indicate that dev-hAM can inhibit inflammation through ECM, growth factors, and/or cytokines; however, int-hAM provides viable endogenous cells that act as an additional source of anti-inflammatory mediators, leading to a higher magnitude of effect than with devitalized tissues.

Clinically, Grafix ${ }^{\circledR}$ (commercially available inthAM) has proved to be effective when used for the treatment of chronic wounds, including chronic diabetic foot ulcers (DFUs), venous leg ulcers, and other surgical wounds. ${ }^{39,40}$ Regulski et al. reported for these wound types an overall $76.1 \%$ wound closure by week 12 . The majority of the wounds in this study had failed other advanced wound-care modalities. ${ }^{39}$ In a 20-center, randomized, controlled, blinded, clinical trial, Grafix was used for the treatment of chronic DFUs. The study showed a statistically significant difference in wound closure at week 12 between the Grafix group and the standard of care group: $62 \%$ versus $21 \%$, respectively $(p=0.0001){ }^{40}$

One limitation of this study, however, is that all data were obtained from in vitro experiments, and it is still unknown to what extent these outcomes are predictive of in vivo responses. Future animal models and clinical trials will help establish this correlation.

\section{INNOVATION}

For wound treatment, hAM has many beneficial properties, including anti-inflammatory, anti-fibrotic, and anti-microbial. Currently, more than 25 commercial hAM products are available. The majority of these products are devitalized with varying degrees 
of structural tissue damage. We recently developed a cryopreservation method that maintains all tissue components, including viable cells, in their native state. In this study, int-hAM showed significantly higher anti-inflammatory activity compared with dev-hAM, which does not contain viable endogenous cells. This work underlies the importance of preserving viable endogenous cells, in addition to structural matrix and growth factors, on the anti-inflammatory activity of hAM.

\section{KEY FINDINGS}

- Fresh hAM can be preserved in its native state with a retained cell viability of $>80 \%$.

- Retention of all tissue components in their native state is essential for achieving a high level of anti-inflammatory activity in cryopreserved hAM.

- In comparison to dev-hAM that contains no viable cells, int-hAM, in which both the structural and cellular integrity of placental tissue is preserved, showed a significantly higher (1) downregulation of proinflammatory TNF- $\alpha$ and IL-1 $\alpha$, (2) upregulation of anti-inflammatory IL-10 and PGE2, and (3) inhibition of collagenase.

\section{ACKNOWLEDGMENTS AND FUNDING SOURCES}

The authors would like to thank Dr. Malathi Sathyamoorthy for her technical support and comments and Ms. Heather Schessler, Dr. Angela Miele, Dr. Jon Hopper, and Mr. Frank Czworka for their valuable comments in reviewing this article. This research was supported by Osiris Therapeutics, Inc.

\section{AUTHOR DISCLOSURE AND GHOSTWRITING}

All listed authors are employees of Osiris Therapeutics, Inc. This article was not written by any writer other than the authors.

\section{ABOUT THE AUTHORS}

Yi Duan-Arnold, PhD, is currently a senior scientist at Osiris Therapeutics, Inc. (OTI). She obtained her $\mathrm{PhD}$ in Biomedical Engineering from City College of New York followed by a postdoctoral training at Yale Medical School. After that, she worked at the Laboratory for Stem Cells and Tissue Engineering at Columbia University on cardiac tissue engineering. Alexandra Gyurdieva, MS, is a scientist at OTI. Amy Johnson, BS, is a senior research associate at OTI. Thomas Uveges, PhD, is an associate director, Orthopaedics at OTI. Douglas Jacobstein, MD, was the senior medical director at OTI when this research was conducted. Currently, he holds a position at Thermo Fisher Scientific, Inc. Alla Danilkovitch, PhD, is currently the Vice President Research and Development at OTI. Dr. Danilkovitch has more than 25 years of a broad biomedical research experience, including stem cell biology, immunology, and cancer research. Dr. Danilkovitch earned her PhD in cell biology.

\section{REFERENCES}

1. Mast BA, Schultz GS. Interactions of cytokines, growth factors, and proteases in acute and chronic wounds. Wound Repair Regen 1996;4 $411-420$

2. Davis JW. Skin transplantation with a review of 550 cases at the Johns Hopkins Hospital. Johns Hopkins Med J 1910;15:307.

3. Akle CA, Adinolfi M, Welsh Kl, Leibowitz $\mathrm{S}$, McColl I. Immunogenicity of human amniotic epithelial cells after transplantation into volunteers. Lancet 1981;2:1003-1005.

4. Robson MC, Duke WF, Krizek TJ. Rapid bacterial screening in the treatment of civilian wounds. $J$ Surg Res 1973;14:426-430.

5. Niknejad, H, Peirovi H, Jorjani M et al. Properties of the amniotic membrane for potential use in tissue engineering. Eur Cell Mater 2008;15:88-99.
6. Adds PJ, Hunt CJ, Dart JK. Amniotic membrane grafts, "fresh" or frozen? A clinical and in vitro comparison. Br J Ophthalmol 2001;85:905-907.

7. Rodriguez-Ares MT, Lopez-Valladares MJ, Tourino $\mathrm{R}$, et al. Effects of lyophilization on human amniotic membrane. Acta Ophthalmol 2009;87:396403.

8. Thomasen $H$, Pauklin M, Steuhl KP, Meller D. Comparison of cryopreserved and air-dried human amniotic membrane for ophthalmologic applications. Graefes Arch Clin Exp Ophthalmol 2009;247:1691-1700

9. Cooke M, Tan EK, Mandrycky C, He H, O'Connell J, Tseng SC. Comparison of cryopreserved amniotic membrane and umbilical cord tissue with dehydrated amniotic membrane/chorion tissue. J Wound Care 2014;23:465-474, 476.
10. Kubo M, Sonoda Y, Muramatsu R, Usui M. Immunogenicity of human amniotic membrane in experimental xenotransplantation. Invest Ophthalmol Vis Sci 2001;42:1539-1546.

11. Rama P, Giannini R, Bruni A, Gatto C, Tiso R, Ponzin D. Further evaluation of amniotic membrane banking for transplantation in ocular surface diseases. Cell Tissue Bank 2001;2:155-163.

12. Kruse FE, Joussen AM, Rohrschneider $K$, et al. Cryopreserved human amniotic membrane for ocular surface reconstruction. Graefes Arch Clin Exp Ophthalmol 2000;238:68-75.

13. Amniox Medical, Inc. CRYOTEK process. www.amnioxmedical.com/CRYOTEK-process.html (last accessed January 19, 2015).

14. Yan G, Sun H, Wang F, et al. Topical application of hPDGF-A-modified porcine BMSC and 
keratinocytes loaded on acellular HAM promotes the healing of combined radiation-wound skin injury in minipigs. Int $\mathrm{J}$ Radiat Biol 2011;87:591600.

15. Maxson S, Lopez EA, Yoo D, Danilkovitch-Miagkova A, Leroux MA. Concise review: role of mesenchymal stem cells in wound repair. Stem Cells Transl Med 2012;1:142-149.

16. Jiang N, Tan NS, Ho B, Ding JL. Azocoll protease activity assay. Protocol Exchange 2007 [Epub ahead of print]; DOl: 10.1038/nprot.2007.484.

17. Bourne $\mathrm{G}$. The Foetal membranes a review of the anatomy of normal amnion and chorion and some aspects of their function. Postgrad Med J 1962; 38:193-201.

18. Fukuda K, Chikama T, Nakamura M, Nishida T. Differential distribution of subchains of the basement membrane components type IV collagen and laminin among the amniotic membrane, cornea, and conjunctiva. Cornea 1999;18:73-79.

19. Parolini O, Alviano F, Bagnara GP, et al. Concise review: isolation and characterization of cells from human term placenta: outcome of the first international Workshop on Placenta Derived Stem Cells. Stem Cells 2008;26:300-311.

20. Sebbag M, Parry SL, Brennan FM, Feldmann M. Cytokine stimulation of $\mathrm{T}$ Iymphocytes regulates their capacity to induce monocyte production of tumor necrosis factor-alpha, but not interleukin10: possible relevance to pathophysiology of rheumatoid arthritis. Eur J Immunol 1997;27:624-632.

21. Harris SG, Padilla J, Koumas L, Ray D, Phipps RP. Prostaglandins as modulators of immunity. Trends Immunol 2002;23:144-150.

22. Silini A, Parolini O, Huppertz B, Lang I. Soluble factors of amnion-derived cells in treatment of inflammatory and fibrotic pathologies. Curr Stem Cell Res Ther 2013;8:6-14.

23. Simpson KL, Keelan JA, Mitchell MD. Labour-associated changes in the regulation of production of immunomodulators in human amnion by glucocorticoids, bacterial lipopolysaccharide and proinflammatory cytokines. J Reprod Fertil 1999;116: 321-327.

24. Eming SA, Krieg T, Davidson JM. Inflammation in wound repair: molecular and cellular mechanisms. J Invest Dermatol 2007;127:514-525.

25. Tan EK, Cooke M, Mandrycky C, et al. Structural and biological comparison of cryopreserved and fresh amniotic membrane tissues. J Biomater Tissue Eng 2014;4:379-388.

26. Liu T, Zhai $H, X u Y$, et al. Amniotic membrane traps and induces apoptosis of inflammatory cells in ocular surface chemical burn. Mol Vis 2012; 18:2137-2146.

27. Rossi D, Pianta S, Magatti M, Sedlmayr P, Parolini 0 . Characterization of the conditioned medium from amniotic membrane cells: prostaglandins as key effectors of its immunomodulatory activity. PLoS One 2012;7:e46956.

28. Hao Y, Ma DH, Hwang DG, Kim WS, Zhang F. Identification of antiangiogenic and antiinflammatory proteins in human amniotic membrane. Cornea 2000;19:348-352.

29. Trengove NJ, Stacey MC, MacAuley S, et al. Analysis of the acute and chronic wound environments: the role of proteases and their inhibitors. Wound Repair Regen 1999;7:442-452.

30. Herrick S, Ashcroft G, Ireland G, Horan M, McCollum C, Ferguson M. Up-regulation of elastase in acute wounds of healthy aged humans and chronic venous leg ulcers are associated with matrix degradation. Lab Invest 1997;77:281288.

31. Grinnell F, Ho CH, Wysocki A. Degradation of fibronectin and vitronectin in chronic wound fluid: analysis by cell blotting, immunoblotting, and cell adhesion assays. J Invest Dermatol 1992;98:410416.

32. Lauer G, Sollberg S, Cole M, et al. Expression and proteolysis of vascular endothelial growth factor is increased in chronic wounds. J Invest Dermatol 2000;115:12-18.

33. Schreml S, Szeimies RM, Prantl L, Karrer S, Landthaler M, Babilas P. Oxygen in acute and chronic wound healing. Br J Dermatol 2010;163: 257-268.

34. Litwiniuk M, Bikowska B, Niderla-Bielinska J, et al. Potential role of metalloproteinase inhibitors from radiationsterilized amnion dressings in the healing of venous leg ulcers. Mol Med Rep 2012; 6:723-728.

35. Wlaschek M, Peus D, Achterberg V, Meyer-Ingold W, Scharffetter-Kochanek K. Protease inhibitors protect growth factor activity in chronic wounds. Br J Dermatol 1997;137:646.

36. King AE, Kelly RW, Sallenave JM, Bocking AD, Challis JR. Innate immune defences in the human uterus during pregnancy. Placenta 2007;28:10991106.

37. Ashcroft GS, Lei K, Jin W, et al. Secretory leukocyte protease inhibitor mediates non-redundant functions necessary for normal wound healing. Nat Med 2000;6:1147-1153.

38. Sorensen OE, Cowland JB, Theilgaard-Monch $\mathrm{K}$, Liu L, Ganz T, Borregaard N. Wound healing and expression of antimicrobial peptides/polypeptides in human keratinocytes, a consequence of common growth factors. J Immunol 2003;170:5583-5589.

39. Regulski M, Jacobstein DA, Petranto RD, Migliori VJ, Nair G, Pfeiffer D. A retrospective analysis of a human cellular repair matrix for the treatment of chronic wounds. Ostomy Wound Manage 2013; 59:38-43.

40. Lavery LA, Fulmer J, Shebetka KA, et al. The efficacy and safety of Grafix $((\mathrm{R}))$ for the treatment of chronic diabetic foot ulcers: results of a multicentre, controlled, randomised, blinded, clinical trial. Int Wound J 2014;11:554-560.

$\begin{aligned} & \text { Abbreviations and Acronyms } \\ & \text { bFGF }=\text { basic fibroblast growth factor } \\ & \mathrm{CD}=\text { cluster of differentiation } \\ & \text { Dev-hAM }=\text { devitalized cryopreserved human } \\ & \quad \text { amniotic membrane } \\ & \mathrm{DFU}=\text { diabetic foot ulcer } \\ & \mathrm{ECM}=\text { extracellular matrix } \\ & \mathrm{EGF}=\text { epidermal growth factor } \\ & \mathrm{HA}=\text { hyaluronic acid } \\ & \mathrm{hAM}=\text { human amniotic membrane } \\ & \mathrm{HGF}=\text { hepatocyte growth factor } \\ & \mathrm{hPBMCS}=\text { human peripheral blood } \\ & \quad \text { mononuclear cells } \\ & \mathrm{IGFBP}=\text { insulin-like growth factor binding } \\ & \text { protein } \\ & \mathrm{IL}=\text { interleukin } \\ & \mathrm{Int}-\mathrm{hAM}==\text { viable intact cryopreserved human } \\ & \quad \text { amniotic membrane } \\ & \mathrm{LPS}=\text { lipopolysaccharide } \\ & \mathrm{MMP}=\text { matrix metalloproteinase } \\ & \mathrm{N}-\mathrm{Gal}=\text { neutrophil gelatinase-associated } \\ & \quad \text { lipocalin } \\ & \mathrm{PDGF}=\text { platelet-derived growth factor } \\ & \mathrm{PGE} 2=\text { prostaglandin E2 } \\ & \mathrm{TGF}=\text { transforming growth factor } \\ & \mathrm{TIMP}=\text { tissue inhibitor of metalloproteinase } \\ & \mathrm{TNF}-\alpha=\text { tumor necrosis factor- } \alpha \\ & \mathrm{VEGF}=\text { vascular endothelial growth factor } \\ &\end{aligned}$

